\title{
Electronic Entanglement in the Vicinity of a Superconductor
}

\author{
Gordey B. Lesovik ${ }^{a, b, c}$, Thierry Martin ${ }^{b}$, and Gianni Blatter ${ }^{c}$ \\ ${ }^{a}$ L.D. Landau Institute for Theoretical Physics, Russian Academy of Sciences, Kosygina Str. 2, 117940, Moscow \\ ${ }^{b}$ Centre de Physique Théorique et Université de la Méditerranée, Case 90\%, 13288 Marseille, France \\ ${ }^{c}$ Theoretische Physik, ETH-Hönggerberg, CH-8093 Zürich, Switzerland
}

(November 6, 2018)

\begin{abstract}
A weakly biased normal-metal-superconductor junction is considered as a potential device injecting entangled pairs of quasi-particles into a normal-metal lead. The two-particle states arise from Cooper pairs decaying into the normal lead and are characterized by entangled spin- and orbital degrees of freedom. The separation of the entangled quasi-particles is achieved with a fork geometry and normal leads containing spin- or energy-selective filters. Measuring the current-current cross-correlator between the two normal leads allows to probe the efficiency of the entanglement cond-mat/0009193.
\end{abstract}

PACS 03.67.Hk,72.70.+m,74.50.+r

The nonlocal nature of quantum mechanics has been demonstrated theoretically [1] using entangled pairs of particles several decades ago. Recently, potential applications of this entanglement have been found in quantum cryptography [2], in quantum teleportation [3], and in quantum computing 肺. It is thus necessary to search for practical ways to produce such pairs given a specific interaction between particles. While past experiments have focused on pairs of photons [5] propagating in vacuum, attention is now turning to electronic systems [6], where this entanglement interaction can be stronger while coherence can still be maintained over appreciable distances in mesoscopic conductors. A scheme was recently presented [7] which discussed the entanglement of electrons via the exchange interaction in pairs of quantum dots. Here, we propose a rather robust electronic entanglement scheme based on the Andreev reflection of electrons and holes at the boundary between a normal metal and a superconductor.

The basic concept underlying the microscopic description of superconductivity is the formation of Cooper pairs. A normal metal in vicinity to a superconductor bears the trace of this phenomenon through the presence of Bogoliubov quasi-particles, or through the non-vanishing of the Gor'kov Green function [8] $F=$ $\left\langle c_{\mathbf{k} \uparrow} c_{-\mathbf{k} \downarrow}\right\rangle$ ( $c_{\mathbf{k} \sigma}$ denote the usual electron annihilation operators). While in a superconductor $F=\Delta / \lambda$ is a consequence of a nonzero gap parameter $\Delta$ ( $\lambda$ is the pairing potential), the coherence surviving in the adjacent normal metal can be understood through the presence of evanescent Cooper pairs. These involve two electrons with entangled spin- and orbital degrees of freedom, carrying opposite spins in the case of usual $s$-wave pairing and with kinetic energies above and below the superconductor chemical potential. This proximity effect has been illustrated in several recent experiments [9].

In order to detect this entanglement and implement it for applications, it is necessary to achieve a spatial sepa- ration between the two constituent electrons. The entanglement apparatus which is proposed here consists of a mesoscopic normal-metal-superconductor (NS) junction with normal leads arranged in a fork geometry (see Fig. 1). Using appropriate spin- or energy-selective filters in the two normal leads the quasi-particle pairs are properly separated and their entanglement can be quantified through a comparison of the intra- and inter- lead noise.

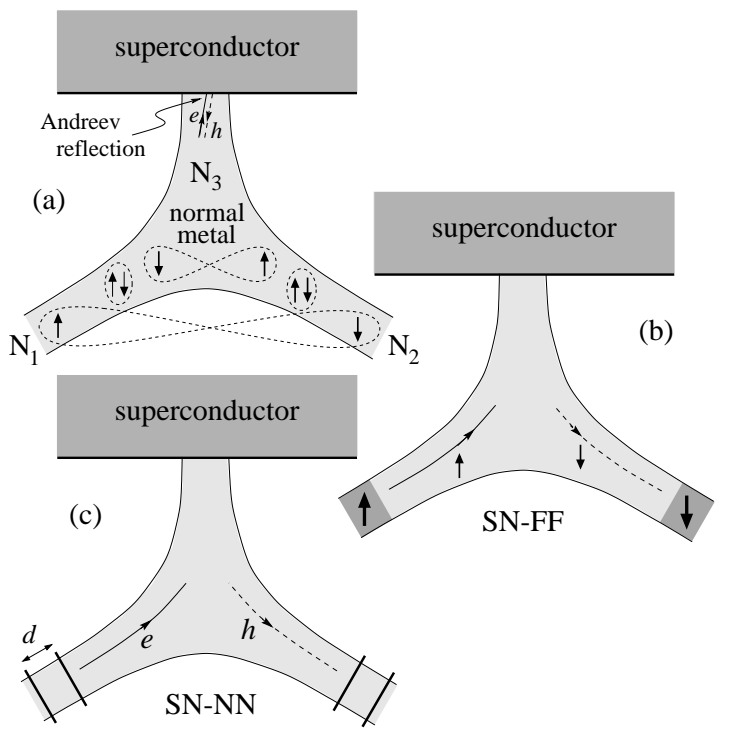

FIG. 1. Normal-metal-superconductor (NS) junction with the normal-metal lead arranged in a fork geometry. (a) Without filters, entangled pairs of quasi-particles (Cooper pairs) injected into the lead $\mathrm{N}_{3}$ propagate into leads $\mathrm{N}_{1}$ or $\mathrm{N}_{2}$ either as a whole or one by one. The ferromagnetic filters in setup (b) enforce the separation of the entangled spins, while the Fabry-Perot type interferometers in the setup (c) separate electron- and hole type quasi-particles.

We start by noting that for a single channel NS wire the zero frequency fluctuations of the currents carried by electrons with different spins are completely correlated,

$$
\left\langle\left\langle I_{\sigma} I_{-\sigma}\right\rangle\right\rangle_{\omega=0} \equiv \int d t\left\langle\left\langle I_{\sigma}(t) I_{-\sigma}(0)\right\rangle\right\rangle=\left\langle\left\langle I_{\sigma}^{2}\right\rangle\right\rangle_{\omega=0},
$$


hence $\left\langle\left\langle\left(I_{\sigma}-I_{-\sigma}\right)^{2}\right\rangle\right\rangle_{\omega=0}=0(\langle\langle\ldots\rangle\rangle$ implies the subtraction of the average currents). This perfect correlation in the (subgap) motion of the quasi-particles with different spins is a consequence of the entanglement of the Cooper pairs injected into the normal wire.

Next, recall that the current noise cross-correlations in a SN-NN fork geometry - without filters on the normal probes - are positive when the transmission between the superconductor and the normal leads is low 10]. The unusual sign (for fermions) of these correlations is due to paired electrons penetrating the two normal leads separately, c.f., Fig. 1(a). The positive correlations are further enhanced when the competing channel (with paired electrons entering the leads jointly) is suppressed through the addition of appropriate spin- or energy selective filters to the normal leads, see Figs. 1(b) and (c). The wave function of the entangled states generated with ideal spin/energy filters then is of the type

$$
\left|\Phi_{\varepsilon, \sigma}^{\mathrm{ent}}\right\rangle=\alpha|\varepsilon, \sigma ;-\varepsilon,-\sigma\rangle+\beta|\mp \varepsilon \pm \sigma ; \pm \varepsilon \mp \sigma\rangle,
$$

where the first (second) argument in $\left|\phi_{1} ; \phi_{2}\right\rangle$ refers to the quasi-particle state in lead 1 (2) evaluated behind the filters and the upper (lower) signs refer to the setup projecting the spin (energy); the coefficients $\alpha$ and $\beta$ can be tuned by external parameters, e.g., a magnetic field. In such a multi-terminal device, the measurement of the zero frequency noise cross-correlator $\left\langle\left\langle I_{\sigma 1} I_{-\sigma 2}\right\rangle\right\rangle_{\omega=0}$ then serves to detect electron entanglement, in analogy with the above single channel NS wire.

A step like dependence of the gap parameter at the NS interface is assumed, subgap transport is specified, while the normal leads are single-channel and ballistic. Using the scattering formulation of NS transport [11], the current operator per spin in normal lead $n$ is defined as

$$
\begin{aligned}
I_{\sigma n}(t) & =\frac{i e \hbar}{2 m} \sum_{\alpha, \alpha^{\prime}} \int_{0}^{\infty} d \varepsilon d \varepsilon^{\prime}\left\{\left[u_{\varepsilon \alpha}^{*}(x) \overleftrightarrow{\partial}_{x} u_{\varepsilon^{\prime} \alpha^{\prime}}(x)\right] \gamma_{\varepsilon \alpha}^{\dagger} \gamma_{\varepsilon^{\prime} \alpha^{\prime}}\right. \\
& \left.-\left[v_{\varepsilon \alpha}^{*}(x) \overleftrightarrow{\partial}_{x} v_{\varepsilon^{\prime} \alpha^{\prime}}(x)\right] \gamma_{\varepsilon^{\prime} \alpha^{\prime}} \gamma_{\varepsilon \alpha}^{\dagger}\right\} \exp \left[i\left(\varepsilon-\varepsilon^{\prime}\right) t\right], \quad
\end{aligned}
$$

where the operators $\gamma_{\varepsilon \alpha}$ describe electron and hole Bogoliubov quasiparticles (with positive energies $\varepsilon$ ) on the normal side with $\alpha=\{p, \sigma, n\}$ a multi-index characterizing the 'charge' $p(=e, h)$, spin $\sigma(= \pm 1 / 2)$, and incidence (lead $n) ; f \stackrel{\leftrightarrow}{\partial}_{x} g \equiv f \partial_{x} g-g \partial_{x} f$. The associated wave functions $\left(u_{\varepsilon \alpha}(x)\right.$ and $\left.v_{\varepsilon \alpha}(x)\right)$ are expressed in terms of the scattering matrix $s_{\alpha, \alpha^{\prime}}$; e.g., for an electron with spin $\sigma$ incident from lead $n$ and observed in lead $m$ at $x_{m}$

$$
\begin{aligned}
& u_{\varepsilon e \sigma n}\left(x_{m}\right) \simeq\left[\delta_{n m} e^{i k_{+} x_{n}}+s_{e \sigma n, e \sigma m} e^{-i k_{+} x_{m}}\right] / \sqrt{h v_{+}}, \\
& v_{\varepsilon e \sigma n}\left(x_{m}\right) \simeq\left[s_{e \sigma n, h-\sigma m} e^{i k_{-} x_{m}}\right] / \sqrt{h v_{-}}
\end{aligned}
$$

with wave numbers $k_{ \pm}=\sqrt{2 m\left(\mu_{\mathrm{S}} \pm \varepsilon\right)}$ and the quasiparticle velocities $v_{ \pm}=\hbar k_{ \pm} / m$ ( $\mu_{\mathrm{S}}$ is the chemical potential in the superconductor). The difference between the two wave numbers $k_{ \pm}$will be neglected $\left(\mu_{\mathrm{S}} \gg \Delta\right)$.
Let us now turn to the fork geometry of Fig. 1: the current leads $N_{1}$ and $N_{2}$ are connected to $N_{3}$, which itself is terminated with the NS interface. We discuss two schemes: a) two ferromagnetic metal contacts (with magnetizations in opposite directions) in leads $N_{1}$ and $\mathrm{N}_{2}$ block the propagation of the opposite spin, see Fig. 1(b), b) two energy filters in $N_{1}$ and $N_{2}$ (coherent quantum dots) select the kinetic energies of electrons and holes symmetrically above and below the superconducting chemical potential (Fig. 1(c)). In both proposals, the penetration of a Cooper pair into a given lead is prohibited, while allowing the split pair to pass the filters. E.g., one electron propagates through $N_{1}$ with spin 'up', while simultaneously the other electron (with opposite kinetic energy) propagates through $N_{2}$ with spin 'down'.

The scattering matrix $s_{\alpha, \alpha^{\prime}}$ has to account for all multiple scattering processes: the Andreev reflection at the $\mathrm{N}_{3} \mathrm{~S}$ interface can be specified in terms of the transmission and reflection amplitudes $t_{13}, t_{23}$, and $r_{i i}(i=$ 1,2 ) describing the normal-metal part of the device [12]. The latter include the scattering by the 'beam splitter' $\mathrm{N}_{3} \longleftrightarrow \mathrm{N}_{1}, \mathrm{~N}_{2}$, and account for the presence of spin or energy filters in leads $\mathrm{N}_{1}$ and $\mathrm{N}_{2}$. The corresponding transmission and reflection amplitudes are found iteratively following the scheme sketched in Fig. 2 and accounting for all interference processes in the device.

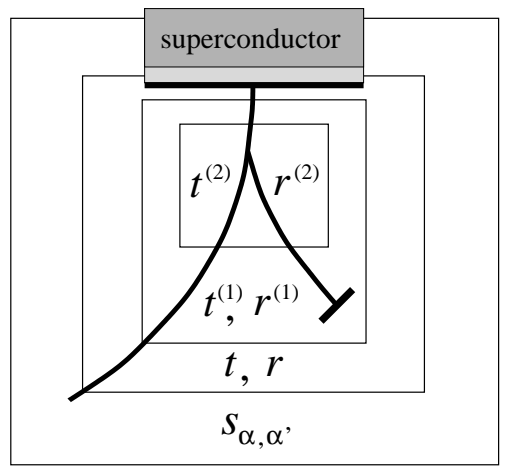

FIG. 2. The scattering matrix $s_{\alpha, \alpha^{\prime}}$ determining the noise correlator (10) incorporates all the internal scattering features of the fork, the beam splitter $\left(\rightarrow t^{(2)}, r^{(2)}\right)$, the stubs $\left(\rightarrow t^{(1)}, r^{(1)}\right)$, and the normal scattering at the NS interface $(\rightarrow t, r)$.

Beam Splitter: Time reversal invariance is assumed for simplicity. It is then possible to express the transmission probability, say, between leads $\mathrm{N}_{1}$ and $\mathrm{N}_{2}$ in terms of the other two transmissions:

$$
T_{12}^{(2)}=T_{13}^{(2)} T_{23}^{(2)}\left[2-T_{\Sigma}^{(2)} \pm 2\left(1-T_{\Sigma}^{(2)}\right)^{1 / 2}\right] / T_{\Sigma}^{(2)}{ }^{2},
$$

where $T_{\Sigma}^{(2)} \equiv T_{13}^{(2)}+T_{23}^{(2)}$, and $T_{i j}^{(2)}=\left|t_{i j}^{(2)}\right|^{2}$. For a fully symmetric beam splitter $T_{i j}^{(2)}=4 / 9$. Ref. [13] focused on a setup which is symmetric between 1and 2 . The lower 
sign in (5) allows to consider also the case where all transmissions are small, thus a more complete parametrization is obtained. The reflection amplitudes of the splitter are

$$
r_{i i}^{(2)}=t_{j k}^{(2) *} t_{i j}^{(2)} t_{i k}^{(2)}\left[T_{j k}^{(2)-1}-T_{i j}^{(2)-1}-T_{i k}^{(2)}{ }^{-1}\right] / 2 .
$$

The phases of the reflection and transmission amplitudes, in addition to describing the intrinsic properties of the links between the three wires, account for the choice of the origin in each lead. Practical choices for these origins are: a) the position of the NS interface in lead $\mathrm{N}_{3}$ and b) that of the filters in $\mathrm{N}_{1}$ and $\mathrm{N}_{2}$. Typical phases accumulated during free propagation are $\exp \left( \pm i k_{ \pm} b\right)$ with $b$ a typical length in the beam splitter.

Stubs: When blocking the propagation through $\mathrm{N}_{2}$ with an ideal filter, the transmission and reflection amplitudes $t_{13}^{(1)}, r_{33}^{(1)}$, and $r_{11}^{(1)}$ follow from the transmission and reflection amplitudes $t_{12}^{(2)}, t_{13}^{(2)}, t_{23}^{(2)}, r_{11}^{(2)}$, and $r_{22}^{(2)}$ of the three bare leads via

$$
\begin{aligned}
& t_{13}^{(1)}=t_{13}^{(2)}-\left(t_{12}^{(2)} t_{23}^{(2)}-t_{13}^{(2)} r_{22}^{(2)}\right) /\left(1+r_{22}^{(2)}\right), \\
& r_{i i}^{(1)}=r_{i i}^{(2)}-t_{2 i}^{(2) 2} /\left(1+r_{22}^{(2)}\right) \quad, \quad(i=1,3)
\end{aligned}
$$

When blocking occurs at $N_{1}$, the amplitudes are obtained by exchanging the lead indices in (7a and (7b).

$N S$ boundary: The NS boundary is split into two parts describing normal and Andreev scattering (Fig. 2). In order to include the normal scattering component the two scatterers $\left\{t_{13}^{(1)}, r_{33}^{(1)}, r_{11}^{(1)}\right\}$ and $\left\{t_{\mathrm{NS}}, r_{\mathrm{NS}}, r_{\mathrm{NS}}^{\prime}\right\}$ are combined to obtain the next level amplitudes:

$$
\begin{aligned}
& t_{13}=t_{13}^{(1)} t_{\mathrm{NS}} /\left(1-r_{33}^{(1)} r_{\mathrm{NS}}^{\prime}\right), \\
& r_{33}=r_{\mathrm{NS}}+t_{\mathrm{NS}}^{2} r_{33}^{(1)} /\left(1-r_{33}^{(1)} r_{\mathrm{NS}}^{\prime}\right), \\
& r_{11}=r_{11}^{(1)}+t_{13}^{(1) 2} r_{\mathrm{NS}}^{\prime} /\left(1-r_{33}^{(1)} r_{\mathrm{NS}}^{\prime}\right),
\end{aligned}
$$

where the primed reflection amplitude $r_{\mathrm{NS}}^{\prime}$ is associated with the particle incident from the superconductor. Finally, we account for perfect Andreev reflection at the NS interface; for an electron incident from $\mathrm{N}_{1}$ the scattering amplitude reads

$$
\left|s_{e \sigma 1, h-\sigma 2}\right|^{2}=\frac{\left|t_{13+}\right|^{2}\left|t_{23-}\right|^{2}}{1+\left|r_{11+}\right|^{2}\left|r_{22-}\right|^{2}+2 \operatorname{Re}\left(r_{33+} r_{33-}^{*}\right)},
$$

where the indices \pm indicate that the energy dependent scattering amplitudes have to be evaluated at the positive(negative) value of the quasi particle energy $\varepsilon(-\varepsilon)$. For each energy $\varepsilon$ only one of the two leads, $\mathrm{N}_{1}$ or $\mathrm{N}_{2}$, is open, resulting in a two-terminal device, thus the relations $\left|r_{i i}\right|^{2}=1-\left|t_{i 3}\right|^{2}=\left|r_{33}\right|^{2}$ hold. The main feature contained in Eq. (9) are the Andreev type resonances building up within the normal-metal leads. These resonances are determined through the sign changes in $\operatorname{Re}\left(r_{33+} r_{33-}^{*}\right)$ and their distance $\sim \hbar v_{\mathrm{F}} / L$ is determined via the Fermi velocity $v_{\mathrm{F}}$ and the characteristic size $L$ of the region. In addition, zeros appear in the spectral density which are a consequence of a vanishing transmission for electrons or holes in this three lead geometry.
The above scheme fully specifies the scattering matrix $s_{\alpha, \alpha^{\prime}}$ for the case with ideal filters. For non-ideal filters the stub should be replaced with a proper description of the lead $\mathrm{N}_{2}$ including its non-ideal filter; in addition, the normal scatterer described through the amplitudes $\left\{t_{13}, r_{33}, r_{11}\right\}$ above has to be combined with an additional scatterer describing the non-ideal filter in the lead $\mathrm{N}_{1}$. E.g., an energy filter requires inclusion of a Fabry-Perot interferometer characterized through the scattering amplitudes $t_{1}, t_{2}, r_{1}^{\prime}, r_{2}$ and the separation $d$ of the double barrier system and producing a transmission $t_{\mathrm{FP}}=t_{1} t_{2} \exp (i k d) /\left[1-r_{1} r_{2}^{\prime} \exp (2 i k d)\right]$. The resonance spacing should be larger than the applied bias for a proper device operation as a filter. The initial resonance lines produced by the quantum dot will then be decorated by Andreev-type resonances and zeros originating from the NS-fork structure.

The above entangler is essentially a two terminal device where electrons with, say, a given spin from lead 1 are converted into holes with an opposite spin in lead 2. The current correlations between 1 and 2 are positive and can be obtained using the definition of the noise in combination with (3), at $T=0$,

$$
\left\langle\left\langle I_{\sigma 1} I_{-\sigma 2}\right\rangle\right\rangle=\frac{e^{2}}{h} \sum_{\alpha, \alpha^{\prime}} \int_{0}^{e V_{1}} d \varepsilon\left|s_{\alpha, \alpha^{\prime}}\right|^{2}\left(1-\left|s_{\alpha, \alpha^{\prime}}\right|^{2}\right),
$$

where a voltage $e V_{1}$ is applied between the lead $\mathrm{N}_{1}$ and the superconductor while keeping the lead $\mathrm{N}_{2}$ unbiased. For the case of ferromagnetic filters, the chemical potential which enters the ( tion functions depends also on the spin index. The multiindices $\alpha$ and $\alpha^{\prime}$ to be summed over in (10) depend on the type of filters in the normal leads $\mathrm{N}_{1}$ and $\mathrm{N}_{2}$ : For ferromagnetic filters (SN-FF) with the spin in $\mathrm{F}_{1(2)}$ pointing up (down) $\alpha=\{e(h) \uparrow 1\}$ and $\alpha^{\prime}=\{h(e) \downarrow 2\}$ (the propagation of other states is blocked by the filters). On the other hand, for the setup selecting a definite quasi particle energy via Fabry-Perot type filters we have to sum over spins with $\alpha=\{e \uparrow(\downarrow) 1\}$ and $\alpha^{\prime}=\{h \downarrow(\uparrow) 2\}$ (we assume filters selecting quasi particles and quasi holes in leads $\mathrm{N}_{1}$ and $\mathrm{N}_{2}$, respectively). Applying the same voltage to the lead $\mathrm{N}_{2}$ as well does not change the answer in the normal fork (SN-NN) but renders the result for the ferromagnetic filters (SN-FF) twice larger. The current fluctuations (10) are straightforwardly converted into "counting" correlations as known from quantum optics: with $e n_{\sigma n}(t)=\int_{0}^{t} d t^{\prime} I_{\sigma n}\left(t^{\prime}\right)$ we find:

$$
\left.\left\langle\left\langle n_{\sigma 1}(t) n_{-\sigma 2}(t)\right\rangle\right\rangle\right|_{t \rightarrow \infty} \approx\left(t / e^{2}\right)\left\langle\left\langle I_{\sigma 1} I_{-\sigma 2}\right\rangle\right\rangle_{\omega=0} .
$$

and hence $\left\langle\left\langle\left(n_{\sigma 1}-n_{-\sigma 2}\right)^{2}\right\rangle\right\rangle /\left\langle\left\langle n_{\sigma 1}^{2}\right\rangle\right\rangle \approx 0$. The result (10) together with the fact that the two currents $I_{\sigma 1}$ and $I_{-\sigma 2}$ are necessarily correlated constitutes the main justification for the proposed entanglement device: Eq. (10) 
corresponds precisely to the current noise in lead 1 . Ideally, these correlation measurements should be performed using fast electronics in order to generate time resolved voltage pulses for electron injection/detection.

This electronic entanglement apparatus can now be completed with a detection apparatus in order to test non-local correlations (Bell inequalities). For an entangler based on ferro/energy filters, the detection apparatus involves filters of opposite type (energy/ferro). Concentrating on energy filters, a positive energy particle emerging in lead $\mathrm{N}_{1}$ can have either spin orientation, which can be "measured" by connecting this lead to, say, a magnetic contact with known spin orientation. In the opposite arm one should have a similar contact but with a magnetization axis rotated by $\pi / 2$ in order to achieve the analog of the spin correlation experiments of Ref. [5]. A detailed discussion of these will be provided later [14.

The proximity induced entanglement of quasi particles in NS-fork type devices was implicit in Refs. [15] and Ref. [16. Consider the above SN-NN setup with the lead $\mathrm{N}_{1}$ biased with respect to the superconductor, while keeping the lead $\mathrm{N}_{2}$ at the superconductor chemical potential. A finite current $I_{2}\left(V_{1}\right)=(2 e / h) \int_{0}^{e V_{1}} d \varepsilon\left|s_{e \uparrow 1, h \downarrow 2}\right|^{2}$ will flow through lead $\mathrm{N}_{2}$ in response to the bias $e V_{1}$ across lead $\mathrm{N}_{1}$. While both experiments in Ref. [15] use a magnetic field to separate electron- and hole type quasi particles, the more recent suggestion in Ref. [16] proposes two ferromagnetic needles, a setup similar to our SNFF device. This Andreev drag effect is quite robust and decreases only gradually with decreasing quality of the filters. The condition for this drag effect to persist reads: $\int_{0}^{e V_{1}} d \varepsilon\left[\left|s_{e \uparrow 1, h \downarrow 2}\right|^{2}-\left|s_{e \uparrow 1, e \uparrow 2}\right|^{2}\right]>0$, implying that the normal current injected from lead $\mathrm{N}_{1}$ to lead $\mathrm{N}_{2}$ remains smaller than the 'drag current' due to Andreev reflected holes. Note that the current $I_{2}$ will vanish when replacing the $s$-wave superconductor with a $p$-wave material or a normal conductor.

Summarizing, we have proposed an electronic entanglement device based on the proximity effect and have shown how to probe the resulting non-local electronic correlations in an emphatic way through a measurement of the current cross-correlator. Using a special fork geometry with, say, Fabry-Perot filters one arrives at a natural source of spin-entangled electron pairs, a device with potential applications in quantum computing architectures based on spintronics [17]. This device presents the advantage - as compared to its ferromagnetic cousin - that it can be realized with nowadays splitters 18 and quantum dot technology, e.g., using semiconductorsuperconductor heterostructures [19], and does not require interdot coupling. Moreover, this SN-NN device appears to be more promising regarding potential applications for quantum information processing: the insertion of Fabry-Perot filters destroys only the orbital en- tanglement of the electrons, while the (most valuable) spin entanglement persists, contrary to the situation in the SN-FF device where the filters project the spin, but where the entanglement of energy degrees of freedom persists nevertheless.

We thank M. Feigelman and M. Reznikov for useful discussions and the Swiss NSF for financial support. GBL acknowledges support from a NWO grant for collaboration with Russia.

[1] A. Einstein, B. Podolsky, and N. Rosen, Phys. Rev. 47, 777 (1935).

[2] A. Ekert, Phys. Rev. Lett. 67, 661 (1991).

[3] C.H. Bennett et al., Phys. Rev. Lett. 70, 1895 (1993).

[4] A. Steane, Rep. Prog. Phys. 61, 117 (1998).

[5] J.F. Glauser and A. Shimony, Rep. Prog. Phys. 41, 1981 (1978), and references therein; A. Aspect, P. Grangier, and G. Roger, Phys. Rev. Lett. 47, 460 (1981); see P.G. Kwiat et al., ibid. 75, 4337 (1995) for modern sources of entangled photon pairs.

[6] G. Burkard, D. Loss, and E. Sukhorukov, Phys. Rev. B 61, R16303 (2000).

[7] D. Loss and E.V. Sukhorukov, Phys. Rev. Lett. 84, 1035 (2000); P. Recher, E.V. Sukhorukov, and D. Loss, condmat/0009452.

[8] A.A. Abrikosov, L.P. Gor'kov, and I.E. Dzyaloshinski, Methods of Quantum Field Theory in Statistical Physics (Prentice-Hall, Englewood Cliffs, N.J., 1963).

[9] V.T. Petrashov et al., Phys. Rev. Lett. 70, 347 (1993); ibid. 74, 5268 (1995); A. Dimoulas et al., ibid. 74, 602 (1995); H. Courtois et al., ibid. 76, 130 (1996); F.B. Müller-Allinger et al., ibid. 84, 3161 (2000).

[10] J. Torrès and T. Martin, Europhys. J. B 12, 319 (1999).

[11] M.J.M. de Jong and C.W.J. Beenakker, Phys. Rev. B 49, 16070 (1994); B.A. Muzykantskii and D.E. Khmelnitskii, ibid. 50, 3982 (1994); M.P. Anantram and S. Datta, ibid. 53, 16390 (1996); G. Lesovik, T. Martin, and J. Torrès, ibid. 60, 11935 (1999).

[12] G. B. Lesovik, A.L. Fauchère, and G. Blatter, Phys. Rev. B 55, 3146 (1997); A.L. Fauchère, G.B. Lesovik, and G. Blatter, ibid. 58, 11177 (1998).

[13] Y. Gefen, J. Imry, and R. Landauer, Phys. Rev. Lett. 52, 139 (1984); M. Büttiker, Y. Imry, and M. Ya. Azbel, Phys. Rev. A 30, 1982 (1984).

[14] G. Blatter, N. Chtchelkatchev, G. B. Lesovik and T. Martin (in preparation).

[15] S.I. Bozhko, V.S. Tsoi and S.E. Yakovlev, Pis'ma Zh. Eksp. Teor. Fiz. 36, 123 (1982) [JETP Lett. 36, 153 (1982)]; P.A.M. Benistant, H. van Kampen and P. Wyder, Phys. Rev. Lett. 51, 817 (1983).

[16] G. Deutscher and D. Feinberg, App. Phys. Lett. 76, 487 (2000).

[17] D. Loss and D.P. di Vincenzo, Phys. Rev. A 57, 120 (1998).

[18] M. Henny et al., Science 284, 296 (1999); W. Oliver et al., ibid., 299 (1999).

[19] H. Takayanagi et al., Phys. Rev. Lett. 75, 3533 (1995). 